\title{
Fumaric aciduria
}

INSERM

\section{Source}

INSERM. (1999). Orphanet: an online rare disease and orphan drug data base. Fumaric aciduria. ORPHA:24

Fumaric aciduria (FA), an autosomal recessive metabolic disorder, is most often characterized by early onset but non-specific clinical signs: hypotonia, severe psychomotor impairment, convulsions, respiratory distress, feeding difficulties and frequent cerebral malformations, along with a distinctive facies. Some patients present with only moderate intellectual impairment. 